\title{
Las metodologías de enseñanza implementadas en la formación inicial docente en Instituciones de Educación Superior de la zona occidental de El Salvador
}

\author{
Eugenia de los Ángeles Repreza \\ Maestría en Asesoría Educativa \\ Docente Investigadora \\ Universidad Católica de El Salvador, El Salvador \\ Email:eugenia.repreza@catolica.edu.sv \\ Recepción: 16-09-2015 / Aceptación: 02-11-2015
}

\section{Resumen}

Esta investigación de tipo explicativo se desarrolló con el propósito de analizar las metodologías de enseñanza implementadas en la formación inicial docente en las Instituciones de Educación Superior de la Zona Occidental de El Salvador, así como su funcionabilidad. Para ello se aplicó la técnica de la entrevista a los docentes de las tres universidades que formaron parte del estudio.

El estudio reflejó que los docentes determinan qué métodos, qué técnicas y qué recursos aplicar, según los objetivos que se le presenten. Esto se refleja en el nivel de atención y participación de los estudiantes; aclarando que la efectividad de esto no sólo depende de la parte metodológica del proceso, sino también de factores - sean estos académicos, personales y sociales -, los cuales en algunos estudiantes les impiden alcanzar el 100\% de los objetivos planteados.

Palabras clave: Metodología, métodos, técnicas, recursos, enseñanza, formación inicial docente.

\begin{abstract}
This explanatory research type was developed in order to analyze teaching methodologies implemented in initial teacher training in higher education institutions of the Western Zone of El Salvador, as well as their functionality. The interview technique was applied to the teachers of the three universities that were part of the study.

The study showed that the teachers determine what methods, what techniques and what resources apply, according to the objectives that are submitted. This is reflected in the level of attention and participation of students; They clarified that the effectiveness of this depends not only on the methodological part of the process, but also of factors - they can be academic, personal and social - which in some students prevent them reaching $100 \%$ of the stated objectives .
\end{abstract}

Key Words: Methodology, method, techniques, resources, teaching, initial teacher training 


\section{Introducción}

La formación inicial docente es entendida como: "un proceso en el que se articulan prácticas de enseñanza y de aprendizaje orientadas a la configuración de sujetos docentes/enseñantes.» (Bonafé, 2001, p. 2); además Rodríguez, citado por García, la entiende como: «la enseñanza profesionalizadora para la enseñanza» (García, 1999, p. 1). Por tanto se debe buscar la formación de sujetos competentes, contribuyendo a la construcción de la sociedad.

En las Normas para la Práctica Docente se considera la formación como «un factor fundamental para fortalecer la educación superior y contribuir a mejorar la calidad en los diferentes niveles del Sistema Educativo $\mathrm{Na}$ cional» (Ministerio de Educación, 1999).

La formación del profesorado debe responder a varios principios, entre ellos el continuo, donde el desarrollo profesional docente es un aprendizaje constante que nunca termina; además de la necesidad de actualización, innovación, cambio y desarrollo curricular. Es por ello que «la investigación es fundamental para poder desarrollar las mejores condiciones que faciliten el aprendizaje de los profesores»(García, 1999, p. 2),, en tanto el proceso de enseñanza-aprendizaje asegure la construcción de aprendizajes significativos (Carretero, 1997, p. 1).

En función de este planteamiento surgió la inquietud de cómo se está abordando la formación inicial de docentes en términos me- todológicos, siendo esto una pieza clave para el alcance de las competencias de los estudiantes, y así poder enfrentar su futuro profesional.

La tarea formativa debe apuntar a la formación de capacidades para elaborar e instrumentar estrategias mediante la capacidad crítica y la actitud filosófica; tendiendo puentes de contenido entre la teoría y la práctica, en un proceso de apropiación de una nueva forma de significar. La teoría debe servir para corregir, comprobar y transformar la práctica, en interrelación dialéctica, fundante de una nueva praxis, que reutiliza la dimensión teórica del conocimiento como base de la acción sustentada (Gorodokin, 2005).

«La formación docente tiene un lugar importante en los procesos de innovación educativa» (Ministerio de Educación, 1997), por tanto es necesario reflexionar sobre el trabajo que hasta el momento a nivel de formación inicial docente se está desarrollando, específicamente en aspectos metodológicos.

«La metodología de la Enseñanza es considerada como la rama de la Pedagogía que utiliza métodos adecuados para la transmisión de conocimientos» (SERCAP, 1993, p. 9). A su vez, para el desarrollo de la metodología, es necesaria la aplicación de técnicas, procedimientos y recursos.

El método es considerado como el «Conjunto de acciones estructuradas, en mayor o menor grado, conforme a procedimientos, formas verbales y modos docentes para la enseñanzaaprendizaje de una disciplina, apropiadas a 
ciertos logros, en espacios y tiempo determinados, con los recursos pertinentes» (Molero, 2004, p. 412). Es por ello que en relación a las metodologías se consideraron como parte de este estudio los métodos, técnicas y recursos.

Entre los métodos de enseñanza colectiva se tienen: Expositivo, de Caso, Interrogativo, de la Lectura, de los Problemas. Además de ello se hace énfasis en el método inductivo y deductivo.

Existen dos tipos de clasificación de técnicas de grupo: las discentes y las docentes. Entre las técnicas discentes se encuentran el Roleplaying (Juego de roles), Torbellino de ideas (Lluvia de ideas), Debate dirigido (discusión guiada), Pequeño grupo de discusión, Phillips $6 / 6$, etc. Entre las técnicas docentes están: el Simposio, la Mesa Redonda, el Panel, Diálogo o Debate público, entre otros. Para el desarrollo de metodologías en el proceso educativo es necesario el uso de diversos recursos, los cuales deberán ser seleccionados y aplicados de manera adecuada.

Definitivamente es importante estar abiertos a valorar el trabajo que otras Instituciones de Educación Superior (IES) realizan, rescatando así lo positivo que puede beneficiar el quehacer docente.

La investigación se desarrolló en las IES de la Zona Occidental de El Salvador encargadas de formar a docentes, sea profesorado o licenciatura: Universidad de Sonsonate (USO), Universidad de El Salvador (UES) y Universidad Católica de El Salvador (UNICAES).
En función de esto, la investigación da respuesta a la siguiente interrogante: ¿Son funcionales las metodologías implementadas en la formación inicial docente en las Instituciones de Educación Superior de la Zona Occidental de El Salvador?

El estudio se dirigió específicamente a responder a los siguientes objetivos específicos, los cuales incluyen las variables principales abordadas: a) Establecer el nivel de formación de los docentes en metodología aplicables en la formación inicial de docentes; b) Puntualizar las metodologías implementadas en la formación inicial de docentes, y c) Determinar la funcionabilidad de las metodologías implementadas en la formación inicial de docentes.

\section{Metodología}

Esta investigación fue de tipo explicativo, ya que analizó la efectividad de las metodologías aplicadas en la formación inicial docente por parte de las IES en la Zona Occidental de El Salvador, brindando razones de la realidad encontrada en el contexto.

Para ello se abordó a una muestra de veinticuatro catedráticos, distribuidos entre las tres universidades de la Zona Occidental de El Salvador que se encargan de la formación inicial de docentes. A continuación se presenta una tabla en la cual se visualiza la distribución de docentes por institución: 
Tabla 1. Distribución de docentes dentro de la Universidades de estudio

\begin{tabular}{|l|c|}
\hline \multicolumn{1}{|c|}{ Universidad } & Cantiad de docentes \\
\hline $\begin{array}{l}\text { Facultad Multidis- } \\
\text { ciplinaria de Occi- } \\
\text { dente - FMO (UES) }\end{array}$ & 6 \\
\hline $\begin{array}{l}\text { Universidad de } \\
\text { Sonsonate (USO) }\end{array}$ & 9 \\
\hline $\begin{array}{l}\text { Universidad Cató- } \\
\text { lica de El Salvador } \\
\text { (UNICAES) }\end{array}$ & 9 \\
\hline Total & 24 \\
\hline
\end{tabular}

La técnica de la entrevista estructurada se les aplicó a los docentes formadores, pertenecientes a las IES. Se utilizó una guía de entrevista, la cual incluyó preguntas en su mayoría abiertas. Las opiniones de los docentes fueron registradas mediante grabación digital.

La investigación se llevó a cabo durante cuatro diferentes etapas, las cuales fueron: Planificación de la investigación, trabajo de campo, Procesamiento, tabulación e interpretación de la información y divulgación de la investigación.

El análisis de la información recolectada se hizo mediante el software Microsoft Excel, organizando los datos en función de las categorías e indicadores establecidos; además se seleccionó este programa por la facilidad que brinda en el manejo de información cuantitativa y cualitativa.

\section{Resultados}

3.1 Sobre formación de docentes en Metodologías aplicables en la formación inicial de docentes

\section{a) Tipo de formación}

Del cien por ciento de los docentes entrevistados, el 91\% afirmó haber recibido formación en cuanto a metodologías aplicables en la formación inicial de docentes; mientras que un $8 \%$ difirió en esta parte. El tipo de formación que han recibido en cuanto a este tema ha sido mediante: la carrera estudiada, cursos, diplomados, talleres y capacitaciones (Ver detalles en tabla 1).

Tabla 2. Tipo de formación de docentes en Metodologías aplicables en la formación inicial de docentes

\begin{tabular}{|l|c|}
\hline \multicolumn{1}{|c|}{ Universidad } & Cantiad de docentes \\
\hline Carrera & 8 \\
\hline Cursos & 7 \\
\hline Diplomado & 5 \\
\hline Taller & 5 \\
\hline Capacitaciones & 3 \\
\hline Ninguno & 2 \\
\hline Total & 30 \\
\hline
\end{tabular}

Fuente: Entrevista a docentes

Las temáticas que afirman haber recibido mediante este tipo de formación son: En mayor medida: a) Metodología para el desarrollo de la Matemática y Lenguaje; b) Metodologías de enseñanza; c) Metodologías activas; d) Aprendizaje cooperativo; e) Métodos de enseñanza y técnicas. En menor medida: a) 
Metodología para la enseñanza de la lectoescritura; b) Metodología para el desarrollo de las ciencias; c) Metodología participativa; d) Metodología por proyectos; e) Metodología sobre inclusión; f) Metodologías para atender a la diversidad; g) Aplicación de métodos inductivos y deductivos; h) Estrategias; i) Metodología para la enseñanza de adultos y $\mathrm{j}$ ) Metodología para la enseñanza en primero y segundo ciclo de Educación Básica.

\section{b) Origen de la formación}

La formación en metodología ha sido recibida, en mayor medida, del Ministerio de Educación (MINED), la Universidad de El Salvador (UES), Fundación Empresarial Para el Desarrollo (ITCA - FEPADE); Universidad José Simeón Cañas (UCA), Universidad de Sonsonate (USO) y de la Editorial Santillana. En menor medida, también consideran que ha provenido de la Universidad Católica de El Salvador (UNICAES), Universidad Francisco Gavidia (UFG), Coordinación Educativa y Cultural Centroamericana (CECC/SICA); Organización de las Naciones Unidas para la Alimentación y la Agricultura (FAO), Organización de Estados Iberoamericanos (OEI), Organización de Estados Americanos (OEA), Escuela Normal; de países como España, Inglaterra, y la Universidad de Bolonia.

\section{c) Suficiencia de formación}

Nótese que si muy bien los docentes manifestaron que en su mayoría han recibido capacitaciones en el tema de metodología, más de la mitad consideran que no es suficiente, aludiendo que siempre se debe aprender y estar en constante actualización (Ver detalles en tabla 2). Los que consideran que sí es suficiente esa formación, afirman tener la fundamentación para hacer la tarea docente, ya que poseen la experiencia por el hecho de trabajar en la especialidad. Solo el 16\% de los docentes afirmó actualizarse mediante la autoformación.

Tabla 3. Suficiencia de formación de docentes en metodologías

\begin{tabular}{|c|c|}
\hline Opción & Frecuencia \\
\hline Sí & 10 \\
\hline No & 14 \\
\hline Total & 24 \\
\hline
\end{tabular}

Fuente: Entrevista a docentes

d) Requerimientos de formación

Ya se ha evidenciado que la mayor parte de los docentes considera que se necesita seguirse actualizando, por lo que las temáticas que plantearon querer recibir:

En mayor medida:

- Tecnología aplicada a la educación: área de laMatemática, Educación Inclusiva, Simposios.

- Metodología para el desarrollo de la Educación Inclusiva y Atención a la diversidad.

En menor medida:

- Metodología en: la enseñanza de la lectoescritura y Ciencias.

- Técnicas de enseñanza 
- Trabajo individual y grupal

- Enseñanza por proyectos

- Manejo de grandes grupos

- Abordaje metodológico de los nuevos programas de estudio del MINED

- Metodología innovadora

- Metodologías activas

- Métodos de enseñanza

- Diferencia entre método, estrategia, técnica y actividades

- Metodología sobre constructivismo y competencias

3.2 Sobre tipos de metodologías implementadas en la formación inicial de docentes
a) Métodos

Entre los métodos que los docentes consideran que aplican en la formación inicial se encuentran: Expositivo, trabajo grupal, trabajo individual, analítico, deductivo, inductivo, estudio de casos, investigativo y participativo (Ver detalles en tabla 4).

Tabla 4. Métodos de enseñanza

\begin{tabular}{|l|c|}
\hline \multicolumn{1}{|c|}{ Opción } & Frecuencia \\
\hline Expositivo & 15 \\
\hline Trabajo grupal & 15 \\
\hline Trabajo individual & 11 \\
\hline Analítico & 10 \\
\hline Deductivo & 9 \\
\hline Inductivo & 8 \\
\hline
\end{tabular}

\begin{tabular}{|l|c|}
\hline \multicolumn{1}{|c|}{ Opción } & Frecuencia \\
\hline Estudio de casos & 8 \\
\hline Investigativo & 6 \\
\hline Participativo & 5 \\
\hline Interrogativo & 3 \\
\hline De la lectura & 3 \\
\hline Método invertido & 2 \\
\hline Método por proyectos & 2 \\
\hline Sintético & 1 \\
\hline Por descubrimiento & 1 \\
\hline De los problemas & 1 \\
\hline Tutoría & 1 \\
\hline Diacrónico & 1 \\
\hline Sincrónico & 1 \\
\hline
\end{tabular}

Fuente: Entrevista a docentes

\section{b) Técnicas}

Entre las técnicas que los docentes aplican en la formación inicial se encuentran: El debate dirigido (discusión guiada), torbellino de ideas (lluvia de ideas), Phillips 6/6, mesa redonda y talleres (Ver detalles en tabla 5).

Tabla 5. Técnicas de enseñanza

\begin{tabular}{|l|c|}
\hline \multicolumn{1}{|c|}{ Opción } & Frecuencia \\
\hline Debate dirigido & 12 \\
\hline Torbellino de ideas & 9 \\
\hline Phillips 6/6 & 4 \\
\hline Mesa redonda & 4 \\
\hline Talleres & 4 \\
\hline El panel & 3 \\
\hline $\begin{array}{l}\text { Aprendizaje } \\
\text { mediante el juego }\end{array}$ & 3 \\
\hline Lectura dirigida & 3 \\
\hline Dramatizaciones & 2 \\
\hline Foros & 2 \\
\hline Recitales & 2 \\
\hline
\end{tabular}




\begin{tabular}{|l|c|}
\hline \multicolumn{1}{|c|}{ Opción } & Frecuencia \\
\hline Dictado & 1 \\
\hline Galerías de arte & 1 \\
\hline Simposio & 1 \\
\hline Pecera & 1 \\
\hline Carrusel & 1 \\
\hline
\end{tabular}

Fuente: Entrevista a docentes

c) Recursos

Entre los recursos que los docentes aplican en la formación inicial se encuentran: Reproductor multimedia, carteles, rotafolio, pizarra, mapas conceptuales, folletos (separatas), videos, plataforma virtual, guías de trabajo y blog (Ver detalles en tabla 6).

Tabla 6. Recursos de enseñanza

\begin{tabular}{|l|c|}
\hline \multicolumn{1}{|c|}{ Opción } & Frecuencia \\
\hline $\begin{array}{l}\text { Reproductor } \\
\text { mutimedia }\end{array}$ & 20 \\
\hline Carteles & 12 \\
\hline Rotafolio & 8 \\
\hline Pizarra & 7 \\
\hline Mapas conceptuales & 7 \\
\hline Folletos, separatas & 7 \\
\hline Videos & 6 \\
\hline Plataforma virtual & 5 \\
\hline Guías de trabajo & 5 \\
\hline Blog & 4 \\
\hline Redes sociales & 3 \\
\hline Internet & 3 \\
\hline Correo electrónico & 3 \\
\hline Libros & 3 \\
\hline Instrumentos de & 3 \\
\hline especialidad & 3 \\
\hline Esquemas & 3 \\
\hline Páginas web & \\
\hline
\end{tabular}

\begin{tabular}{|l|c|}
\hline \multicolumn{1}{|c|}{ Opción } & Frecuencia \\
\hline Diapositivas & 3 \\
\hline Canciones & 2 \\
\hline Ilustraciones & 2 \\
\hline Franelógrafo & 2 \\
\hline Portafolio & 2 \\
\hline Rompecabezas & 2 \\
\hline Textos impresos & 2 \\
\hline Cuadros sinópticos & 2 \\
\hline Recursos del entorno & 2 \\
\hline Tutoriales & 1 \\
\hline Proyector de acetatos & 1 \\
\hline Centro de cómputo & 1 \\
\hline Radiograbadora & 1 \\
\hline Diagramas & 1 \\
\hline Hojas de ejercicios & 1 \\
\hline
\end{tabular}

Fuente: Entrevista a docentes

\subsection{Sobre funcionabilidad de metodo- logías implementadas en la formación inicial de docentes}

a) Tipos de metodologías más efectivas

El 33\% de los docentes consideró que para ellos todos los métodos y técnicas aplicadas les han sido efectivos.

Ellos manifestaron que los métodos que les han sido más efectivos son: Expositivo, Estudio de casos, Investigativo, participativo, trabajo grupal, trabajo individual y analítico. En cuanto a técnicas están: Debate dirigido, talleres, aprendizaje mediante el juego y el torbellino de ideas. Finalmente en cuanto a recursos están: Reproductor multimedia, carteles, internet, diapositivas, rotafolio, videos, plataforma virtual, blog e instrumentos de especialidad. 
b) Funcionabilidad de métodos, técnicas $y$ recursos

Todos los docentes manifestaron que las metodologías implementadas en la formación inicial les han sido funcionales. Específicamente, seis docentes afirmaron que la evaluación evidencia el aprendizaje del estudiante; mientras que cuatro de ellos consideraron que mediante ella se alcanzan los objetivos; y finalmente, dos docentes afirmaron que, a través de la evaluación, se logra que el estudiante se integre. Además otros docentes brindaron diversas razones entre las cuales se encuentran: «Se alcanza éxito en la prueba $\mathrm{ECAP}$ » «las evaluaciones orales lo demuestran»; "permite participación del estudiante en clase»; «les permite detectar deficiencias y realizar nivelación»; «permite adaptación al contenido que se desarrolla»; «son pocos los estudiantes que reprueban»; «los alumnos siguen indicaciones»; "cumplen con actividades», y finalmente, "por la asistencia de los alumnos en clase».

c) Adecuación de las metodologías a lo requerido en los objetivos básicos de las asignaturas

Desde el punto de vista de todos los docentes las metodologías aplicadas se adecuan a lo requerido en los objetivos básicos de las asignaturas (Ver detalles en tabla 7).
Tabla 7. Adecuación de metodología a objetivos de asignaturas

\begin{tabular}{|c|c|}
\hline Opción & Frecuencia \\
\hline Sí & 24 \\
\hline No & 0 \\
\hline Total & 24 \\
\hline
\end{tabular}

Fuente: Entrevista a docentes

Según ellos, consideraron que se alcanza esta adecuación porque: «Los recursos y contenidos están acordes a la realidad laboral futura»; «los estudiantes demuestran entender las temáticas»; $y$ "porque se toman de referencia los objetivos para establecer la metodología». Además siete docentes afirmaron que esa adecuación no se alcanza en un $100 \%$, porque «hace falta tener espacio para modelar con estudiantes en el entorno real»; «hay desinterés de algunos estudiantes», «la acumulación de trabajos de las diferentes asignaturas», "por el tiempo y el espacio», «el alto número de estudiantes por sección», y finalmente, «la reposición de clases a veces no se puede dar».

d) Valoración del alcance del desarrollo de teoría-práctica mediante las metodologías aplicadas

Veintidós docentes consideraron que sí se alcanza el desarrollo de la teoría-práctica mediante las metodologías aplicadas. Un docente manifestó que «Depende», ya que considera que existen inconvenientes como la indisciplina de los estudiantes que afecta e impide el alcance completo. Además un docente opinó que ello «No se alcanza», porque el objetivo está planteado; pero el estudiante muchas ve- 
ces no lo conoce, y en parte el mismo docente a veces no se lo explica.

e) Nivel de atención de estudiantes en el proceso educativo

Todos los docentes manifestaron que la metodología utilizada les permite que los estudiantes presten atención en clases, observándolo mediante las siguientes manifestaciones: Por la participación, las preguntas constantes que realizan a lo largo de la temática; los comentarios coherentes mediante el lenguaje gestual; el involucramiento en actividades grupales; cuando se acercan a pedir orientación, y por la socialización que establecen en clase. Dos docentes dijeron que esta atención no se da al $100 \%$ porque ésta depende del tema que se está desarrollando y de la dosificación del proceso educativo.

\section{f) Nivel de participación del estudiante en el proceso educativo}

El 71\% de los docentes valoró como «Intermedio» el nivel de participación del estudiante en el proceso educativo; a diferencia del 29\% restante que lo calificó como «Alto». Las razones que brindaron al respecto hicieron referencia a que existe irresponsabilidad, apatía, desinterés, timidez y distracción por algunos estudiantes; así como la interferencia que algunos estudiantes generan en clase (Ver detalles en Tabla 8).
Tabla 8. Nivel de participación del estudiante en el proceso educativo

\begin{tabular}{|c|c|}
\hline Opción & Frecuencia \\
\hline Alto & 7 \\
\hline Intermedio & 17 \\
\hline Bajo & 0 \\
\hline Total & 24 \\
\hline
\end{tabular}

Fuente: Entrevista a docentes

g) Fortalecimiento del nivel vocacional del estudiante

Se tomó en consideración como punto clave la «Vocación» para determinar la efectividad de la metodología implementada en la formación inicial docente. Para ello los catedráticos han compartido las estrategias que llevan a cabo para fortalecerla en los estudiantes:

- Se les presenta la realidad que vive un docente.

- Se realiza investigación acción para abordar problemas prácticos.

- Se orienta en cuanto al vocabulario y presentación personal que un docente debe reflejar.

- Se les habla de la ética que el docente debe tener.

- Se trabaja la parte afectiva del estudiante mediante el diálogo.

- Se le plantean casos concretos de lo que se da en la realidad del profesor.

- Incluye reflexiones que les permiten entender para qué se están formando y cómo será su rol como futuros docentes. 
- Se visitan instituciones educativas para que descubran por ellos mismos si tienen vocación o no para la carrera.

- Se trabaja con proyección social.

- Se relaciona el tema con la realidad profesional a la cual se enfrentarán.

h) Rendimiento académico del estudiante en formación docente

El 42\% de los docentes valoró como «Alto» el rendimiento académico de los estudiantes en formación docente; el 54\% como «Intermedio»; y solo el 4\% lo consideró como «Bajo» (Ver Tabla 9).

Tabla 9. Rendimiento académico del estudiante en formación docente

\begin{tabular}{|c|c|}
\hline Opción & Frecuencia \\
\hline Alto & 10 \\
\hline Intermedio & 13 \\
\hline Bajo & 1 \\
\hline Total & 24 \\
\hline
\end{tabular}

Fuente: Entrevista a docentes

Las razones que manifestaron acerca de la valoración realizada es que consideran que los estudiantes: «les cuesta aprender más a unos que a otros», "hay alumnos que trabajan y eso les afecta»; «hay jóvenes sin visión ni objetivos de vida», "no todos los estudiantes se esmeran por igual», y porque «algunos estudiantes vienen con deficiencias en analizar e interpretar».

\section{Discusión}

Es incuestionable que el rol del docente en la calidad educativa es clave, y la toma de decisiones de la metodología que se implementará, es uno de los compromisos principales que recae en sus hombros.

El docente requiere un lugar más protagónico en el discurso de la calidad educativa, dado que es él quien tiene un contacto directo con los estudiantes, con los planes y programas de estudio, así como con la realidad cotidiana de la vida en las aulas, lo que le permite conocer las necesidades de los alumnos. (Chehaybar y Kuri, Edith, 2007, p. 105).

Por esa razón, se analizó la metodología que los docentes de las IES de la Zona Occidental del país, encargadas de la formación inicial docente, aplican; además de la verificar su efectividad.

Es notorio que si bien los docentes poseen formación en ésta área, igual manifiestan la necesidad de seguirse formando, no dando por terminado el proceso de actualización. Según Eric Hoffer «En tiempos de cambio, quienes estén abiertos al aprendizaje se adueñarán del futuro, mientras que aquellos que creen caberlo todo estarán bien equipados para un mundo que no existe». (Económicas, de la Calidad, \& Honduras, s/f). Es necesario entonces que exista una constante preparación del docente en estos temas, permitiéndoles innovar y generar procesos educativos de calidad: todo en pro del beneficio de los estudiantes. 
El Ministerio de Educación de El Salvador (MINED), Universidades y otras instituciones están contribuyendo a la formación del docente en temáticas sobre metodología, lo cual es trascendental dentro de la gestión que a nivel institucional y personal se realiza para acceder a este tipo de capacitaciones. De esta forma, a futuro, se pueden fortalecer los vacíos que los mismos docentes manifestaron tener y que les lleva a interesarse a recibir procesos de formación, como por ejemplo: Metodología en la enseñanza de asignaturas básicas, sobre métodos y técnicas.

Es de enfatizar que los docentes mencionaron que aplican diversidad de métodos para la enseñanza, así como también diferentes técnicas y recursos; lo cual hace ver que éstos están tratando de hacer su parte en la labor docente. Ahora bien, si se observan los resultados generales, se nota esta tendencia; pero si se analizan los resultados de manera individual, es claro que los docentes no están aplicando diversidad metodológica.

En cuanto a los recursos aplicados, se evidencia un escaso uso de libros de textos que sirven como base de las cátedras en la formación inicial docente, haciéndose presente más las separatas o folletos, los cuales son copias parcializadas de los documentos originales. Esto destaca el uso que se está haciendo de las Tecnologías de la Información y la Comunicación (TIC), pero hace falta un mayor aprovechamiento del internet y redes sociales. Los más utilizados como recursos son el reproductor multimedia, los carteles, rotafo- lio, pizarra, mapas conceptuales, folletos, separatas, videos, plataforma virtual, guías de trabajo y blog.

Cada docente valora que los métodos, técnicas y recursos que aplica le han sido funcionales, apuntando específicamente a la adecuación que existe de éstos a los objetivos de la asignatura. Significa entonces que los docentes están determinando qué métodos, qué técnicas y qué recursos aplicarán según los objetivos que se le presenten. Viéndose esta efectividad en el nivel de atención y participación de los estudiantes. Claro está que la efectividad no sólo depende de la parte metodológica del proceso, sino también de otros factores, sean estos académicos, personales y sociales, los cuales en algunos estudiantes les impide alcanzar el 100\% de los objetivos planteados. Esto, a la larga, transforma en el objeto de investigación de futuros estudios. 


\section{Referencias}

Bonafé, J. M. (2001). Arqueología del concepto “compromiso social” en el discurso pedagógico y de formación docente. REDIE: Revista Electrónica de Investigación Educativa, 3(1), 6.

Carretero, M. (1997). ¿Qué es el constructivismo? Recuperado de http://upvv.clavijero.edu. $\mathrm{mx} /$ cursos/SerFacilitadorCambioParadigma/vector2/actividad5/documentos/Constructivismo_Carretero.pdf

Chehaybar y Kuri, Edith. (2007). Reflexiones sobre el papel del docente en la calidad educativa. Redalyc, 50. Recuperado de http://www.redalyc.org/pdf/340/34005013.pdf

Económicas, J. J. Á. M. L. en A. de E. y C., de la Calidad, D. en G., \& Honduras, M. en A. de E. M. de 35 años de experiencia como catedrático de la U. N. A. de. (s/f). Actualización constante como necesidad para el Docente... Recuperado de http://www.gestiopolis.com/actualizacionconstante-como-necesidad-para-el-docente-universitario/

García, P. C. (1999). Formación del profesorado. Barcelona: Praxis. Recuperado de http://observatorio.org/colaboraciones/2003/PROFESORADO\%20-\%20PedroChagoyan\%204ene03.pdf

Gorodokin, I. C. (2005). La formación docente y su relación con la epistemología. Revista iberoamericana de educación, 37(5), 5.

El Salvador, Ministerio de Educación. (s.f.). Ley de la Carrera Docente. San Salvador, El Salvador. El Salvador, Ministerio de Educación. (1994). Ley General de Educación. San Salvador, El Salvador. El Salvador, Ministerio de Educación. (1997). Normas y orientaciones curriculares para la formación inicial de maestros. San Salvador, El Salvador.

El Salvador, Ministerio de Educación. (1999). Normas para la práctica docente: Formación inicial de maestros. San Salvador, El Salvador.

El Salvador, Ministerio de Educación de España. (2004). Investigaciones sobre el inicio de la lectoescritura en edades tempranas. Ministerio de Educación.

SERCAP. (1993). Metodología. SERCAP. 